\title{
Supplementary Information \\ Simultaneous Retrieval of the Size and Refractive Index of Suspended Droplets in a Linear Quadrupole Electrodynamic Balance
}

\author{
Chelsea L. Price ${ }^{1}$, Alison Bain², Brandon J. Wallace ${ }^{2}$, Thomas C. Preston², James F. Davies ${ }^{1 *}$ \\ 1. Department of Chemistry, University of California Riverside, Riverside CA 92420 USA \\ 2. Department of Atmospheric and Oceanic Sciences and Department of Chemistry, McGill University, \\ Montreal, Quebec, Canada \\ *Correspondence to: jfdavies@ucr.edu
}

Spectral Range of Deliquescence Measurements

Figure S1(A-E) shows the spectra used in the analysis of the deliquescence of $\mathrm{NaCl}$ droplets detailed in Table 1. The shaded regions indicate the range that was used in the sizing analysis, chosen due to the possibility for unambiguous identification of pairs of TE and TM modes in a given mode order and number. Other parts of the spectrum show sharp peaks atop broader peaks, and this can cause complications in the peak fitting process leading to a greater level of uncertainty in the results. 

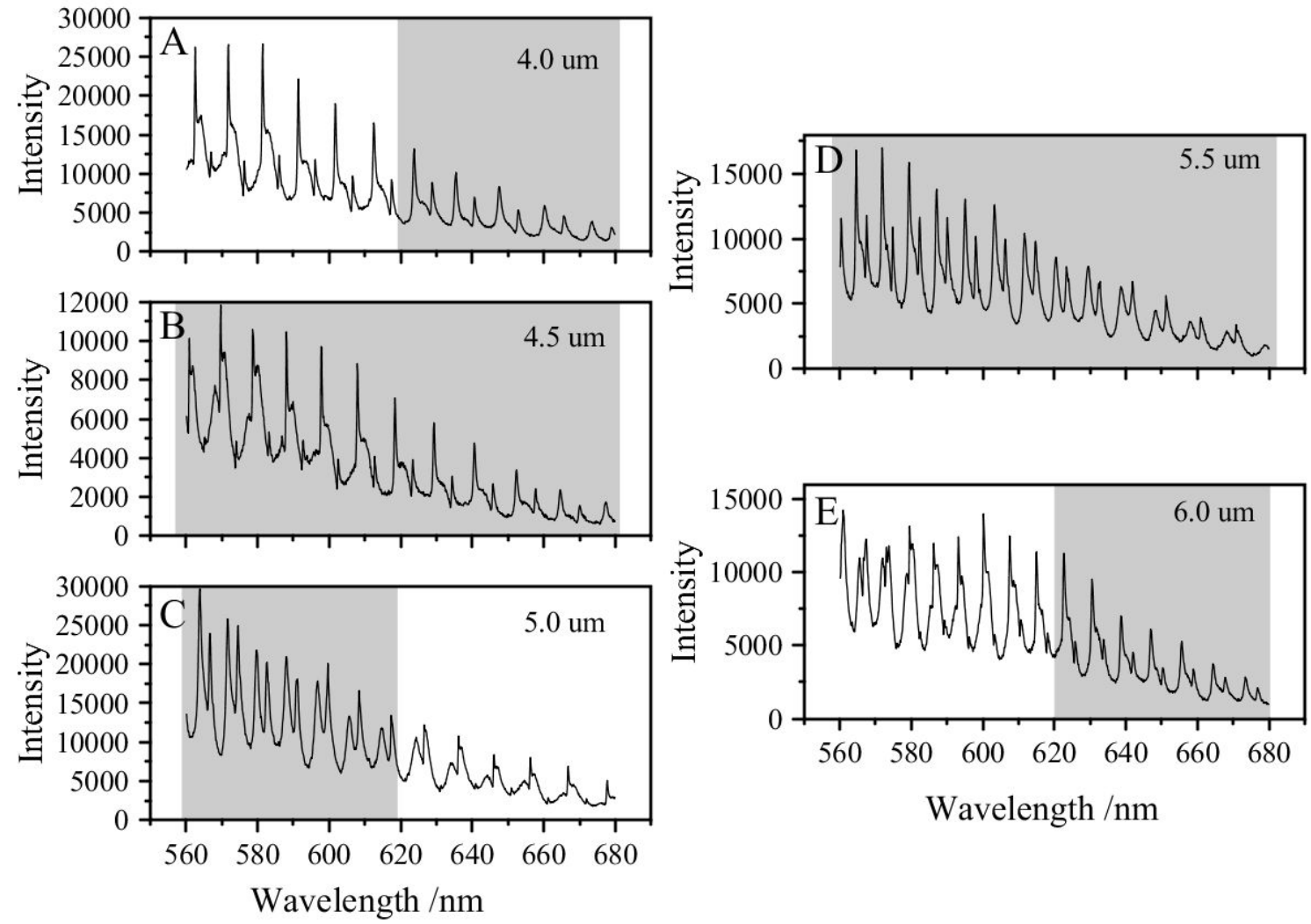

Figure S1: Mie resonance spectra for $\mathrm{NaCl}_{(\mathrm{aq})}$ droplets at the deliquescence $\mathrm{RH}$, indicating the wavelength range used in the fitting process. The results are shown in Table 1.

\section{Heating in Light-Absorbing Droplets}

To verify that the droplet is not significantly perturbed due to heating by LED illumination, a sample of containing equal parts $\mathrm{NaCl}$ and $\mathrm{NaHA}$ was sampled over a range of supply current to the LED, from $0.2 \mathrm{~A}$ to $1.2 \mathrm{~A}$. This varies the light intensity by a factor of $\sim 6$ and the resulting Mie resonance spectra were observed (Figure S2A). Despite the large change in illumination intensity, very little change in the spectrum was observed, indicating the extent of heating does not significantly perturb the liquid-vapor equilibrium of water and no change in size occurs. This is in stark contrast to the effect of laser illumination at $532 \mathrm{~nm}$, which results in a large change in the spectrum for even low power unfocused laser light. 


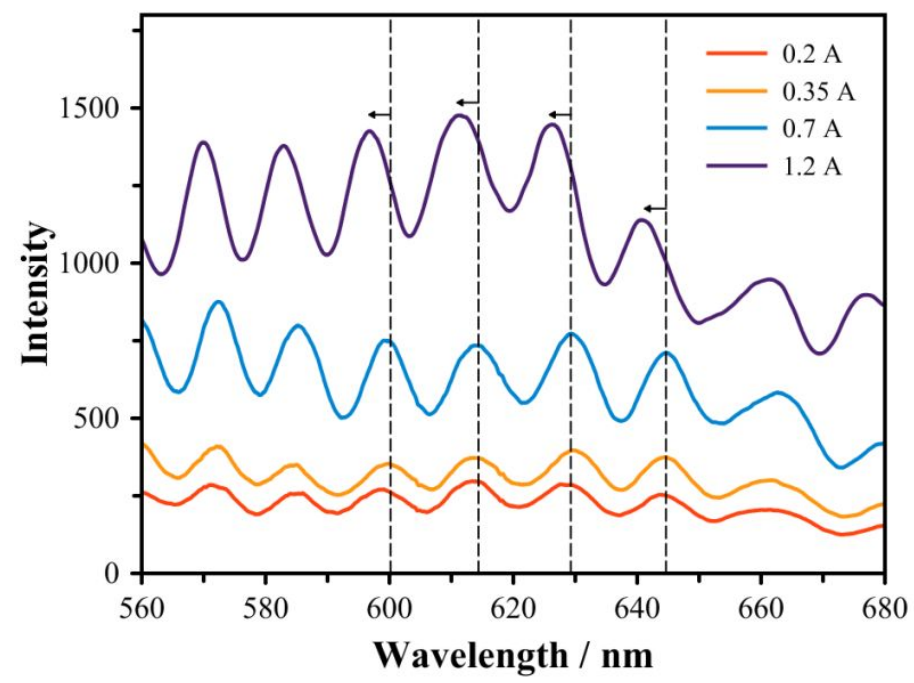

Figure S2: A droplet containing equal parts $\mathrm{NaCl}$ and $\mathrm{NaHA}$ at $75 \% \mathrm{RH}$ was exposed to increasing intensity of light from the white LED illumination source, as represented by the illumination current. Very little change in the (unnormalized) spectrum was observed indicating no significant heating of the droplet. The arrows show a small shift in peak position at $1.2 \mathrm{~A}$, however this is attributed to small changes in the $\mathrm{RH}$ in the chamber rather than heating of the droplet. 